ESAIM: Proceedings, Vol. 4, 1998, 223-233

Contrôle et Équations aux DÉrivées Partielles

http://www.emath.fr/proc/Vol.4/

\title{
DYNAMIC DOMAIN DECOMPOSITION OF OPTIMAL CONTROL PROBLEMS FOR NETWORKS OF EULER-BERNOULLI BEAMS
}

\author{
G. LEUGERING ${ }^{1}$ \\ Institute of Mathematics \\ University of Bayreuth \\ D-95440 Bayreuth \\ Germany \\ leugering@uni-bayreuth.de
}

Key Words : elastic beams, boundary control, dynamic domain decomposition.

AMS Subject Qualification : 49K20, 65P05, 73K05, 73D30, 35Q72, 35B37.

\footnotetext{
${ }^{1}$ This research was supported by the DFG under grant Le595/9-1.
}

Article published by EDP Sciences and available at http://www.edpsciences.org/proc or http://dx.doi.org/10.1051/proc:1998030 


\begin{abstract}
We consider planar networks of Euler-Bernoulli beams subject to Neumanntype boundary controls at simple nodes. The object is to minimize a cost functional along some part of the beam structure by the way of dynamic domain decomposition.
\end{abstract}




\section{Introduction}

We consider a simple planar graph $G=(V, E)$ with vertices $V=\left\{v_{J}, v_{N}, v_{M} \ldots\right\}$ labeled by capital letters, and edges $E=\left\{e_{i}, e_{j}, \ldots\right\}$ labeled by lower case letters. We denote by $e_{i}$ the normalized vector in the direction of the edge $i$. Further, let $d_{J}$ denote the edge degree of the vertex $v_{J}$. Then we can distinguish between multiple nodes $V_{M}$, where the $d_{J}{ }^{\prime} s$ are $>1$, and simple nodes $V_{S}$ with $d_{J}=1$. The simple nodes consist of Dirichlet-nodes $V_{D}$, where the beam is clamped and Neumann-nodes $V_{N} \cup \dot{U} V_{C}$, where the label $N$ signifies a free end, while $C$ signifies a controlled Neumann-node. We denote the deformation of the $i$-th beam by

$$
r_{i}(x, t)=u_{i}(x, t) e_{i}+w_{i}(x, t) e_{i}^{\perp},
$$

where $u_{i}, w_{i}$ represent the longitudinal and vertical displacement in the local coordinate system. Let $\epsilon_{i J}$ be $=1$ if edge $i$ ends at the vertex $v_{J}$, and $=-1$ if it starts there (one may always consider $\epsilon_{i J}=0$ if the $i$-th edge is not incident at $v_{J}$ ). Further, let $\mathcal{E}_{J}$ denote the set of indices belonging to edges incident at $v_{J}$. For more detailed modelling issues we refer to [7]. We first write down the equations governing the motion of the elastic network of Euler-Bernoulli beams. Rayleigh-beams can also be treated similarly.

$$
\begin{aligned}
& \left\{\begin{array}{l}
\rho_{i} \ddot{u}_{i}=E h_{i} u_{i}^{\prime \prime}, \\
\rho_{i} \ddot{w}_{i}+E I_{i} w_{i}^{\prime \prime \prime \prime}=0, \quad \text { in }\left(0, l_{i}\right) \times(0, T),
\end{array}\right. \\
& \left\{\begin{array}{l}
r_{i}\left(v_{J}\right)=r_{j}\left(v_{J}\right), \\
w_{i}^{\prime}\left(v_{J}\right)=w_{j}^{\prime}\left(v_{J}\right), \quad \forall i, j \in \mathcal{E}_{J}, v_{J} \in V_{M},
\end{array}\right. \\
& \left\{\begin{array}{l}
r_{i}\left(v_{D}\right)=0, \\
w_{i}^{\prime}\left(v_{D}\right)=0, \quad \text { if } \epsilon_{i D} \neq 0, v_{D} \in V_{D},
\end{array}\right. \\
& \left\{\begin{array}{l}
\sum_{i \in \mathcal{E}_{J}} \epsilon_{i J} E I_{i} w_{i}^{\prime \prime}\left(v_{J}\right)=0, \\
\sum_{i \in \mathcal{E}_{J}} \epsilon_{i J}\left(E h_{i}, u_{i}^{\prime}\left(v_{J}\right) e_{i}-E I_{i} w_{i}^{\prime \prime \prime}\left(v_{J}\right) e_{i}^{\perp}\right)=f_{J},
\end{array}\right. \\
& r_{i}(\cdot, 0)=r_{i 0}, \dot{r}_{i}(0,0)=r_{J 1} \text { on }\left(0, l_{i}\right) .
\end{aligned}
$$

Here the first set of equations (1.1) represents the dynamics of the longitudinal and vertical displacements along an individual edge $i,(1.2)$ characterizes the continuity of displacements and rotation angles accross the joint $v_{J}$, which is then said to be rigid. Position (1.3) signifies a clamped node, while the two nodal balance laws (1.4) represent the balance of moments and forces at $v_{J}$, respectively, $f_{J}=0$ for $v_{J} \notin V_{C}$. Finally, (1.5) are the initial data for the problem. For definiteness, we assume that $\left|V_{D}\right| \geq 1$.

ESAIM: Proc., Vol. 4, 1998, 223-233 
The problem of exact controllability of (1.1)-(1.5) has been studied in [11] for $G$ being a star-graph with $\left|V_{M}\right|=1, V_{C}=V_{S}$. For non collinear in-plane-beam structures this is, to our knowledge, the only result available in the literature, whereas serial (out-of-the-plane) beams have been widely studied [5], [3], [14], to name just a few. The paper [3] contains rotational inertia of cross sections and joint-masses. While we have been able in [7] to show exact controllability of Timoshenko-tree-like networks, we have not succeeded so far in passing to Euler-Bernoulli or Rayleigh-beam networks. As a result, the controllabilitytheory and hence also the theory of uniform stabilization of such networks is widely open.

This motivates the interest in optimal control problems for beam structures. In this note we give a first attempt towards this goal. The main point is that we want to achieve optimal control of such networks by domain decomposition, i.e. we want to reduce the global optimality system to a collection of local ones, which in turn we might solve in parallel.

We have been able to achieve this goal for networks of strings in [8], [9] and Timoshenko-beams [10] by various methods, where we have been inspired by recent work of Benamou [1], [2], and Glowinski et.al. [4], Quarteroni and Valli [13].

For the sake of simplicity, we consider the following cost functional

$$
J(f):=\frac{1}{2} \sum_{v_{J} \in V_{C}} \int_{0}^{T}\left|f_{J}\right|^{2} d t+\frac{1}{2} \sum_{i=1}^{n_{e}} \int_{0}^{T} \int_{0}^{l_{i}}\left|r_{i}\right|^{2} d x d t,
$$

where $r_{i}$ satisfies (1.1)-(1.5). We note that we can also treat the case, where not all edges are involved in the cost. This gives the possibility of silencing just some edges. Other cost functions involving penalized end conditions are currently under investigation. However, as opposed to [9], more subtle arguments seem to be necessary in the beam case. Functionals like (1.6) have been considered by Benamou for serial planar $2-d$ decompositions without transmission phenomena. Such set-up for transmission problems is the subject of current joint work with J.E. Lagnese.

\section{The global optimality system}

We introduce the adjoint state $p_{i}:=\varphi_{i} e_{i}+\psi_{i} e_{i}^{\perp}$. Then, using standard arguments, we derive the following set of equations describing the backwards running adjoint variables.

$$
\begin{aligned}
& \left\{\begin{array}{l}
\rho_{i} \ddot{\varphi}_{i}=E h_{i} \varphi_{i}^{\prime \prime}+u_{i}, \\
\rho_{i} \ddot{\psi}_{i}+E I_{i} \psi_{i}^{\prime \prime \prime \prime}=w_{i}, \quad \text { on }\left(0, l_{i}\right) \times(0, T),
\end{array}\right. \\
& \left\{\begin{array}{l}
p_{i}\left(v_{J}\right)=p_{j}\left(v_{J}\right), \\
\psi_{i}^{\prime}\left(v_{J}\right)=\psi_{j}^{\prime}\left(v_{J}\right), \quad \forall i, j \in \mathcal{E}_{J}, v_{J} \in V_{M},
\end{array}\right.
\end{aligned}
$$




$$
\begin{aligned}
& \left\{\begin{array}{l}
p_{i}\left(v_{D}\right)=0, \\
\psi_{i}^{\prime}\left(v_{D}\right)=0 \quad \text { if } \epsilon_{i D} \neq 0, v_{D} \in V_{D},
\end{array}\right. \\
& \left\{\begin{array}{l}
\sum_{i \in \mathcal{E}_{J}} \epsilon_{i J} E I_{i} \psi_{i}^{\prime \prime}\left(v_{J}\right)=0, \\
\sum_{i \in \mathcal{E}_{J}} \epsilon_{i J}\left(E h_{i}, \psi_{i}^{\prime}\left(v_{J}\right) e_{i}-E I_{i} \psi_{i}^{\prime \prime \prime}\left(v_{J}\right) e_{i}^{\perp}\right)=0, \\
\forall v_{J} \in V_{M} \cup V_{N} \cup V_{C}, t \in(0, T),
\end{array}\right. \\
& \left\{\begin{array}{l}
p_{i}(T)=0, \\
\dot{p}_{i}(T)=0 \quad \text { on }\left(0, l_{i}\right) .
\end{array}\right. \\
& f_{J}=-p_{i}\left(v_{J}\right), v_{J} \in V_{C}, i \in \mathcal{E}_{C}, t \in(0, T) .
\end{aligned}
$$

\section{Well-posedness}

We introduce the space setting for problems like (1.1)-(1.5), (2.1)-(2.6).

$$
\begin{aligned}
H= & \prod_{i=1}^{n_{e}} L^{2}\left(0, l_{i}\right)^{2} \\
V= & \left\{v \in H \mid v_{i} \cdot e_{i} \in H^{1}\left(0, l_{i}\right), v_{i} \cdot e_{i}^{\perp} \in H^{2}\left(0, l_{i}\right)\right. \\
& v_{i}\left(v_{D}\right)=0, v_{i}^{\prime} \cdot e_{i}^{\perp}\left(v_{D}\right)=0, \epsilon_{i D} \neq 0, v_{D} \in V_{D} \\
& \left.v_{i}\left(v_{J}\right)=v_{j}\left(v_{J}\right), v_{i}^{\prime} \cdot e_{i}^{\perp}\left(v_{J}\right)=v_{j}^{\prime} \cdot e_{j}^{\perp}\left(v_{J}\right), \quad \forall i, j \in \mathcal{E}_{J}, v_{J} \in V_{M}\right\}
\end{aligned}
$$

The space $V$ is a Hilbert space compactly embedded in $H$. Moreover, if $\left|V_{D}\right| \geq 1$, then the total potential energy

$$
E_{p o t}:=\frac{1}{2} \sum_{i=1}^{n_{e}} \int_{0}^{l_{i}} E h_{i} u_{i}^{\prime 2}+E I_{i} w_{i}^{\prime \prime 2} d x
$$

is seen to be equivalent to the norm of the Sobolev space

$$
H^{1} \times H^{2}:=\prod_{i=1}^{n_{e}} H^{1}\left(0, l_{i} ; e_{i}\right) \otimes H^{2}\left(0, l_{i} ; e_{i}^{\perp}\right),
$$

where the appearance of $e_{i}, e_{i}^{\perp}$ indicates longitudinal and vertical parts.

We may now define the operator $A$ in $H$ as follows

$$
\begin{aligned}
A r= & \left(-E h_{i} u_{i}^{\prime \prime} ; E I_{i} w_{i}^{\prime \prime \prime \prime}\right)_{i=1}^{n_{e}} \\
D(A)= & \left\{r \in V \mid r_{i}=u_{i} e_{i}+w_{i} e_{i}^{\perp}, u_{i} \in H^{2}\left(0, l_{i}\right), w_{i} \in H^{4}\left(0, l_{i}\right),\right. \\
& \sum_{i \in \mathcal{E}_{J}} \epsilon_{i J} E I_{i} w_{i}^{\prime \prime}\left(v_{J}\right)=0 \\
& \sum_{i \in \mathcal{E}_{J}} \epsilon_{i J}\left(E h_{i} u_{i}^{\prime}\left(v_{J}\right) e_{i}-E I_{i} w_{i}^{\prime \prime \prime}\left(v_{J}\right) e_{i}^{\perp}\right)=0 \\
& \left.v_{J} \in V_{M} \cup V_{N} \cup V_{C}\right\}
\end{aligned}
$$

ESAIM: Proc., Vol. 4, 1998, 223-233 
We have

Theorem 3.1 A defined by (3.5), (3.6) is self-adjoint and nonnegative. If $V_{D} \neq \emptyset$, then $A$ is positive definite. A has discrete spectrum.

Theorem 3.2 Let $V_{D} \neq \emptyset, f \in \prod_{v_{J} \in V_{C}} L^{2}(0, T)^{2}, r_{0} \in H, r_{1} \in V^{\star}$. Then there exists a unique weak solution $r$ of (1.1)-(1.5) such that $r \in C(0, T, H)$ $\cap C^{1}\left(0, T, V^{\star}\right) \cap C^{2}\left(0, T, D(A)^{\star}\right)$.

Remark i) More regular, i.e. finite energy, strong solutions etc. are obtained in the standard way for more regular data. However, a sharp regularity theory for beam networks of Euler-Bernoulli-type does not seem to be known at this point.

ii) The global optimality system (1.1)-(1.5), (2.1)-(2.5), (2.6) has a unique (weak, mild or strong) solution, according to the regularity of the data.

\section{Domain decomposition}

As an exemplaric situation, we condsider an edge connecting a multiple node $v_{J}$ to a controlled simple node $v_{C}$. The other cases are to be treated in a similar fashion.

In order to derive the decoupling node conditions we utilize the observation made by Glowinski and Le Tallec in [6] that the non-overlapping Schwarziteration considered by P.L. Lions [12] is in fact equivalent to an augmented Lagrangian approach combined with a standard saddle-point algorithm. This observation has lead us to derive dynamic domain decompositions of string and Timoshenko beam networks, see [9], [10]. Because of the limited space, we cannot go into details, but rather present the result. The detailed analysis will be published in a forthcoming paper.

We consider the system on edge $i$ :

$$
\begin{gathered}
\rho_{i} \ddot{u}_{i}^{n+1}-E h_{i}\left(u_{i}^{n+1}\right)^{\prime \prime}=0 \\
\rho_{i} \ddot{w}_{i}^{n+1}+E I_{i}\left(w_{i}^{n+1}\right)^{\prime \prime \prime \prime}=0 \\
\rho_{i} \ddot{\varphi}_{i}^{n+1}-E h_{i}\left(\varphi_{i}^{n+1}\right)^{\prime \prime}=u_{i} \\
\rho_{i} \ddot{\psi}_{i}^{n+1}+E I_{i}\left(\psi_{i}^{n+1}\right)^{\prime \prime \prime \prime}=w_{i}, \\
\epsilon_{i C}\left(E h_{i}\left(u_{i}^{n+1}\right)^{\prime}\left(v_{C}\right) e_{i}-E I_{i}\left(w_{i}^{n+1}\right)^{\prime \prime \prime}\left(v_{C}\right) e_{i}^{\perp}=-p_{i}^{n+1}\left(v_{C}\right),\right. \\
\epsilon_{i C}\left(E h_{i}\left(\varphi_{i}^{n+1}\right)^{\prime}\left(v_{C}\right) e_{i}-E I_{i}\left(\psi_{i}^{n+1}\right)^{\prime \prime \prime}\left(v_{C}\right) e_{i}^{\perp}=0,\right. \\
\epsilon_{i C}\left(E I_{i}\left(w_{i}^{n+1}\right)^{\prime \prime}\left(v_{C}\right)=\epsilon_{i C} E I_{i}\left(\psi_{i}^{n+1}\right)^{\prime \prime}\left(v_{C}\right)=0,\right.
\end{gathered}
$$




$$
\begin{aligned}
& \epsilon_{i J} E h_{i}\left(u_{i}^{n+1}\right)^{\prime}\left(v_{J}\right)+\varphi_{i}^{n+1}\left(v_{J}\right)=\left(\frac{2}{d_{J}} \sum_{j \in \mathcal{E}_{J}} p_{j}^{n}\left(v_{J}\right) e_{i}-\varphi_{i}^{n}\left(v_{J}\right)\right) \\
& -\left(\frac{2}{d_{J}} \sum_{j \in \mathcal{E}_{J}} \epsilon_{j J}\left\{E h_{j}\left(u_{j}^{n}\right)^{\prime}\left(v_{J}\right) e_{j}-E I_{j}\left(w_{j}^{n}\right)^{\prime \prime \prime}\left(v_{J}\right) e_{j}^{\perp}\right\} e_{i}\right. \\
& \left.-\epsilon_{i J} E h_{i}\left(u_{i}^{n}\right)^{\prime}\left(v_{J}\right)\right), \\
& -\epsilon_{i J} E I_{i}\left(w_{i}^{n+1}\right)^{\prime \prime \prime}\left(v_{J}\right)+\psi_{i}^{n+1}\left(v_{J}\right)=\left(\frac{2}{d_{J}} \sum_{j \in \mathcal{E}_{J}} p_{j}^{n}\left(v_{J}\right) e_{i}^{\perp}-\psi_{i}^{n}\left(v_{J}\right)\right) \\
& -\left(\frac{2}{d_{J}} \sum_{j \in \mathcal{E}_{J}} \epsilon_{j J}\left\{E h_{j}\left(u_{j}^{n}\right)^{\prime}\left(v_{J}\right) e_{j}-E I_{j}\left(w_{j}^{n}\right)^{\prime \prime \prime}\left(v_{J}\right) e_{j}^{\perp}\right\} e_{i}\right. \\
& \left.+\epsilon_{i J} E I_{i}\left(w_{i}^{n}\right)^{\prime \prime \prime}\left(v_{J}\right)\right) \\
& \epsilon_{i J} E I_{i}\left(w_{i}^{n+1}\right)^{\prime \prime}\left(v_{J}\right)+\left(\psi_{i}^{n+1}\right)^{\prime}\left(v_{J}\right)=\left(\frac{2}{d_{J}} \sum_{j \in \mathcal{E}_{J}}\left(\psi_{j}^{n}\right)^{\prime}\left(v_{J}\right)-\left(\psi_{i}^{n}\right)^{\prime}\left(v_{J}\right)\right) \\
& -\left(\frac{2}{d_{J}} \sum_{j \in \mathcal{E}_{J}} \epsilon_{j J} E I_{j}\left(w_{j}^{n}\right)^{\prime \prime}\left(v_{J}\right)-\epsilon_{i J} E I_{i}\left(w_{i}^{n}\right)^{\prime \prime}\left(v_{J}\right)\right), \\
& \epsilon_{i J} E h_{i}\left(\varphi_{i}^{n+1}\right)^{\prime}\left(v_{J}\right)-u_{i}^{n+1}\left(v_{J}\right)=-\left(\frac{2}{d_{J}} \sum_{j \in \mathcal{E}_{J}} r_{j}^{n}\left(v_{J}\right) e_{i}-u_{i}^{n}\left(v_{J}\right)\right) \\
& -\left(\frac{2}{d_{J}} \sum_{j \in \mathcal{E}_{J}} \epsilon_{j J}\left\{E h_{j}\left(\varphi_{j}^{n}\right)^{\prime}\left(v_{J}\right) e_{j}-E I_{j}\left(\psi_{j}^{n}\right)^{\prime \prime \prime}\left(v_{J}\right) e_{j}^{\perp}\right\} e_{i}\right. \\
& \left.-\epsilon_{i J} E h_{i}\left(\varphi_{i}^{n}\right)^{\prime}\left(v_{J}\right)\right) \\
& -\epsilon_{i J} E I_{i}\left(\psi_{i}^{n+1}\right)^{\prime \prime \prime}\left(v_{J}\right)-\left(w_{i}^{n+1}\right)\left(v_{J}\right)=-\left(\frac{2}{d_{J}} \sum_{j \in \mathcal{E}_{J}} r_{j}^{n}\left(v_{J}\right) e_{i}^{\perp}-w_{i}^{n}\left(v_{J}\right)\right) \\
& -\left(\frac{2}{d_{J}} \sum_{j \in \mathcal{E}_{J}} \epsilon_{j J}\left\{E h_{j}\left(\varphi_{j}^{n}\right)^{\prime}\left(v_{J}\right) e_{j}-E I_{j}\left(\psi_{j}^{n}\right)^{\prime \prime \prime}\left(v_{J}\right) e_{j}^{\perp}\right\} e_{i}\right. \\
& \left.+\epsilon_{i J} E I_{i}\left(\psi_{i}^{n}\right)^{\prime \prime \prime}\left(v_{J}\right)\right) \\
& \epsilon_{i J} E I_{i}\left(\psi_{i}^{n+1}\right)^{\prime \prime}\left(v_{J}\right)-\left(w_{i}^{n+1}\right)^{\prime}\left(v_{J}\right)=-\left(\frac{2}{d_{J}} \sum_{j \in \mathcal{E}_{J}}\left(w_{j}^{n}\right)^{\prime}\left(v_{J}\right)-\left(w_{i}^{n}\right)^{\prime}\left(v_{J}\right)\right) \\
& -\left(\frac{2}{d_{J}} \sum_{j \in \mathcal{E}_{J}} \epsilon_{j J} E I_{j}\left(\psi_{j}^{n}\right)^{\prime \prime}\left(v_{J}\right)-\epsilon_{i J} E I_{i}\left(\psi_{i}^{n}\right)^{\prime \prime}\left(v_{J}\right)\right),
\end{aligned}
$$

ESAIM: Proc., Vol. 4, 1998, 223-233 


$$
r_{i}(\cdot, 0)=r_{i 0}, \dot{r}_{i}(\cdot, 0)=r_{i 1}, p_{i}(\cdot, T)=\dot{p}_{i}(\cdot, T)=0 .
$$

\section{A local optimal control problem}

It can be shown that the system (4.1)-(4.9) is, in fact, an optimality system for an optimal control problem on the edge $i$ :

$$
\begin{aligned}
\min _{f_{C}, f_{J}, m_{J}} & \frac{1}{2} \int_{0}^{T} \int_{0}^{l_{i}}\left|r_{i}^{n+1}\right|^{2} d x t+\frac{1}{2} \int_{0}^{T}\left\{\left|f_{J}\right|^{2}+\left|f_{C}\right|^{2}+\left|m_{J}\right|^{2}\right\} d t \\
& +\frac{1}{2} \int_{0}^{T}\left\{\left|r_{i}^{n+1}\left(v_{J}\right)+\mu_{i J}^{n}\right|^{2}+\left|\left(w_{i}^{n+1}\right)^{\prime}\left(v_{J}\right)+\eta_{i, J}^{n}\right|^{2}\right\} d t
\end{aligned}
$$

subject to

$$
\begin{aligned}
\rho_{i} \ddot{u}_{i}^{n+1}=E h_{i}\left(u_{i}^{n+1}\right)^{\prime \prime}, \rho_{i} \ddot{w}_{i}^{n+1}+E I_{i}\left(w_{i}^{n+1}\right)^{\prime \prime \prime \prime} & =0 \\
\epsilon_{i C}\left(E h_{i}\left(u_{i}^{n+1}\right)^{\prime}\left(v_{C}\right) e_{i}-E I_{i}\left(w_{i}^{n+1}\right)^{\prime \prime \prime}\left(v_{C}\right) e_{i}^{\perp}\right. & =f_{C}, \\
\epsilon_{i J}\left(E h_{i}\left(u_{i}^{n+1}\right)^{\prime}\left(v_{J}\right) e_{i}-E I_{i}\left(w_{i}^{n+1}\right)^{\prime \prime \prime}\left(v_{J}\right) e_{i}^{\perp}\right) & =f_{J}+\lambda_{i J}^{n}, \\
\epsilon_{i C}\left(E I_{i}\left(w_{i}^{n+1}\right)^{\prime \prime}\left(v_{C}\right)=0, \epsilon_{i J} E I_{i}\left(w_{i}^{n+1}\right)^{\prime \prime}\left(v_{J}\right)\right. & =m_{J}+\sigma_{i J}^{n}, \\
r_{i}^{n+1}=r_{i 0}, \dot{r}_{i}^{n+1} & =r_{i 1} .
\end{aligned}
$$

Here $\lambda_{i J}^{n}=\lambda_{i J}^{n} \cdot e_{i} e_{i}+\lambda_{i J}^{n} \cdot e_{i} e_{i}^{\perp}$ and $\lambda_{i J}^{n} \cdot e_{i}, \lambda_{i J}^{n} \cdot e_{i}^{\perp}, \sigma_{i J}^{n}$ are given by the right hand sides of (4.3)-(4.5). Accordingly, $\mu_{i J}^{n} \cdot e_{i}, \mu_{i J}^{n} \cdot e_{i}^{\perp}, \eta_{i J}^{n}$ are given by the right hand sides of (4.6)-(4.8).

Now, decomposing the initial and boundary data into its local $\left(e_{i}, e_{i}^{\perp}\right)$ coordinates, the optimal control problem (5.1), (5.2) separates in two optimal control problems, one for the longitudinal and one for the vertical displacement. Each of the problems can be shown to possess a unique set of minimizers. In fact, these are quadratic problems with well-posed dynamics for finite energy initial data and $L^{2}$-boundary inputs.

\section{Convergence}

In order to prove convergence of the local pairs, which we call $\left(\hat{r}_{i}^{n}, \hat{p}_{i}^{n}\right)$, satisfying (4.1)-(4.9) (or (5.1), (5.2)) to the local restrictions $\left(r_{i}, p_{i}\right)$ of the global optimality system $(r, p)$ satisfying $(2.1)-(2.6)$, we introduce the errors $\tilde{r}_{i}^{n}=r_{i}-\hat{r}_{i}^{n}, \tilde{p}_{i}^{n}=$ $p_{i}-\hat{p}_{i}^{n}$. It can be seen that $\tilde{r}_{i}^{n}, \tilde{p}_{i}^{n}$ satisfy (4.1)-(4.9) with zero initial data for $\tilde{r}_{i}^{n}$ (and of course zero final data for $\tilde{p}_{i}^{n}$ ).

We define

$$
\begin{aligned}
E^{n+1}:= & \sum_{J} \sum_{i \in \mathcal{E}_{J}} \int_{0}^{T}\left\{\left|E h_{i}\left(\tilde{\varphi}_{i}^{n+1}\right)^{\prime}\left(v_{J}\right)\right|^{2}+\left|E h_{i}\left(\tilde{u}_{i}^{n+1}\right)^{\prime}\left(v_{J}\right)\right|^{2}\right. \\
& +\left|E I_{i}\left(\tilde{\psi}_{i}^{n+1}\right)^{\prime \prime \prime}\left(v_{J}\right)\right|^{2}+\left|E I_{i}\left(\tilde{w}_{i}^{n+1}\right)^{\prime \prime \prime}\left(v_{J}\right)\right|^{2}+ \\
& +\left|E I_{i}\left(\tilde{\psi}_{i}^{n+1}\right)^{\prime \prime}\left(v_{J}\right)\right|^{2}+\left|E I_{i}\left(\tilde{w}_{i}^{n+1}\right)^{\prime \prime}\left(v_{J}\right)\right|^{2}+
\end{aligned}
$$




$$
\begin{aligned}
& +\left|\tilde{r}_{i}^{n+1}\left(v_{J}\right)\right|^{2}+\left|\tilde{p}_{i}^{n+1}\left(v_{J}\right)\right|^{2}+\left|\left(\tilde{\psi}_{i}^{n+1}\right)^{\prime}\left(v_{J}\right)\right|^{2} \\
& \left.+\left|\left(\tilde{w}_{i}^{n+1}\right)^{\prime}\left(v_{J}\right)\right|^{2}\right\} d t .
\end{aligned}
$$

Then it can be shown by massive calculations that the following recursion formula holds

$$
\begin{aligned}
E^{n+1}=E^{n} & -2\left(\sum_{i=1}^{n_{e}} \int_{0}^{T} \int_{0}^{l_{i}}\left|\tilde{r}_{i}^{n+1}\right|^{2} d x d t+\sum_{v_{C} \in V_{C}} \int_{0}^{T}\left|\tilde{p}_{i}^{n+1}\left(v_{C}\right)\right|^{2} d t\right)(6.2) \\
& -2\left(\sum_{i=1}^{n_{e}} \int_{0}^{T} \int_{0}^{l_{i}}\left|\tilde{r}_{i}^{n}\right|^{2} d x d t+\sum_{v_{C} \in V_{C}} \int_{0}^{T}\left|\tilde{p}_{i_{C}}^{n}\left(v_{C}\right)\right|^{2} d t\right) \\
= & E^{0}-2 \sum_{k=0}^{n+1^{\prime}} \sum_{i=1}^{n_{e}} \int_{0}^{T} \int_{0}^{l_{i}}\left|\tilde{r}_{i}^{k}\right|^{2} d x d t-2 \sum_{k=0}^{n+1^{\prime}} \sum_{v_{C} \in V_{C}} \int_{0}^{T}\left|\tilde{p}_{i_{C}}^{k}\left(v_{C}\right)\right|^{2} d t
\end{aligned}
$$

(with $\sum_{i=0}^{n '} a_{i}=\frac{1}{2} a_{0}+\frac{1}{2} a_{n}+\sum_{i=1}^{n-1} a_{i}$ ).

Now, letting $n$ tend to infinity we see that $\left(E^{n}\right)_{n}$ remains bounded, while

:

$$
\sum_{k=0}^{\infty} \sum_{i=1}^{n_{e}} \int_{0}^{T} \int_{0}^{l_{i}}\left|\tilde{r}_{i}^{k}\right|^{2} d x d t<\infty
$$

implies the convergence to zero of the direct error

$$
\int_{0}^{T} \int_{0}^{l_{i}}\left|\tilde{r}_{i}^{k}\right|^{2} d x d t \longrightarrow 0 \quad k \rightarrow \infty \quad \forall i=1, \ldots, n_{e}
$$

and

$$
\sum_{k=0}^{\infty} \sum_{v_{C} \in V_{C}} \int_{0}^{T}\left|\tilde{p}_{i_{C}}^{k}\left(v_{C}\right)\right|^{2} d t<\infty
$$

implies

$$
\int_{0}^{T}\left|\tilde{p}_{i_{C}}^{k}\right|^{2} d t \longrightarrow 0 \quad k \rightarrow \infty \quad \forall i_{C}: \epsilon_{i_{C} C} \neq 0 .
$$

The limiting adjoint system $p=\left(p_{i}\right)_{i}$ can be shown to admit the zero solution only. We can, therefore, show

Theorem 6.1 Let $E^{0}$ be finite. Then the sequence of pairs $\left(\hat{r}_{i}^{n}, \hat{p}_{i}^{n}\right)$ satisfying (4.1)-(4.9) converge to $\left(r_{i}, p_{i}\right)$ such that $(r, p)$ satisfies the global optimality system (2.1)-(2.6). The convergence takes place in $L^{2}(0, T, H)$.

Remark. i) More can be said about the sense of convergence as the boundedness of $\left(E^{n}\right)_{n}$ implies weak- $L^{2}(0, T)$ convergence on subsequences of all traces. We defer also this analysis to a

: forthcoming paper.

ESAIM: Proc., Vol. 4, 1998, 223-233 
ii) We can also consider masses at the joints. We have developed algorithms which iteratively decouple the beams from the motion of the masses, so that the local problems which have to be computed numerically reduce to classical Euler-Bernoulli-problems rather than to hybrid ones.

iii) We currently consider this procedure also for networks of large deflection beams.

iv) As in [1] one can derive local feedback controls by the way of Riccati equations.

v) As to how the approach presented here can be used to study controllability problems is subject to current research.

\section{References}

[1] Benamou, J.-D. (1996), "Analyse numerique - Décomposition de domaine pour le contrôle de systèmes gouvernés par des équations d'evolution", Indiana University Mathematics Journal, Preprint.

[2] Benamou, J.-D. (1995), "A domain decomposition method for the optimal control of systems governed the Helmholtz equation", Mathematical and numerical aspects of wave propagation, (Cohen, G. ed.), SIAM,New York, 653-662.

[3] Castro, C. and Zuazua, E. (1997), "Boundary controllability of a hybrid system consisting in two flexible beams connected by a point mass", to appear.

[4] Bourgat, J. F. and Glowinski, R. and Le Tallec, P. and Vidrascu, M. (1989), "Variational Formulation and Algorithm for Trace Operator in Domain Decomposition Calculations", Domain Decomposition Methods, (Chan, T. and Glowinski, R. and Periaux, J. and Widlund, O. B., Eds.), SIAM, New York.

[5] Chen, G. and Delfour, M. C. and Krall, A. M. and Payre, G. (1987), "Modeling, stabilization and control of serially connected beams", SIAM J. Control and Optimization, Vol.25, 526-546.

[6] Glowinski, P. and Le Tallec, P. (1990), "Augmented Lagrangian interpretation of the nonoverlapping Schwarz alternating method", The Third International Symposium on Domain Decomposition Methods for Partial Differential Equations, ( Chan, T. and Glowinski, R. Eds.), SIAM, New York.

[7] Lagnese, J.E. and Leugering, G. and Schmidt E.J.P.G. (1994), Modeling, Analysis and Control of Dynamic Elastic Multi-Link Structures, Birkhäuser,Boston-Basel-Berlin".

[8] Leugering, G. (1997), "On domain decomposition of controlled networks of elastic strings and joint masses", Control of Distributed Parameter Systems, (Kappel, F., Ed.),to appear. 
[9] Leugering, G. (1997), "A domain decomposition of optimal control problems for dynamic networks of elastic strings", Optimal Control: Theory, Algorithms, and Applications, (Hager, W. W. and Pardalos, P. M. , Eds.), Kluwer Publishers, to appear.

[10] Leugering, G. (1997), "A domain decomposition technique for dynamic networks of Timoshenko beams", to appear.

[11] Leugering, G. and Schmidt, E.J.P.G. (1989), "On the control of networks of vibrating strings and beams", Proceedings of the 28th IEEE Conference on Decision and and Control,IEEE, 3:2287-2290

[12] Lions, P.L. (1990), "On the Schwarz alternating method 3", The Third International Symposium on Domain Decomposition Methods for Partial Differential Equations, (Chan, T. and Glowinski, R., Eds.),SIAM New York.

[13] Quarteroni, A. and Valli, A. (1990), "Theory and application of SteklovPoincaré operators for boundary-value problems. The heterogenous operator case", The Third International Symposium on Domain Decomposition Methods for Partial Differential Equations, (Chan, T. and Glowinski, R., Eds.),SIAM New York.

[14] Taylor, S.W. (1992), "Exact boundary controllability of a beam and mass system", Computation and Control IV, (Bowers, K. L. and Lund, J.,Eds.), Birkhäuser Basel,305-321 